\title{
Sôbre alqumas equações de diferenças relacionadas com a Genética de Populações
}

\author{
FREDERICO PIMENTEL GOMES \\ Professor Substituto de Matemática da E. S. A. \\ "Luiz de Queiroz"
}

INDICE

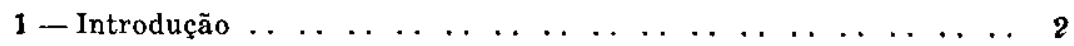

2 - Um problema típico de Genética de Populações .. . . . . . 2

3- Um problema típico de probobilidades . . . . . . . . . . . . .

4 - Como se aplica o método das funções geratrizes ... . . . 5

5 - Mais um exemplo de aplicação frequente $\ldots \ldots \ldots$

6 - Bibliografia consultada . . . . . . . . . . . . . . . . . . . . . . 11 


\section{1 - INTRODUÇÃO}

A aplicação das equações de diferenças ao Cálculo de Probabilidades é bem antiga. Mas na Genética de Populaçóes, que é de origem recente, as equações de diferenças têm um papel fundamental, donde a necessidade em que se encontram os geneticistas de estudar os métodos que nos permitem resolvê-las.

Nêste trabalho examinaremos a resolução das equações lineares de diferenças pelo método das funções geratrizes, introduzido por P.. S. Laplace (1749-1827) e extensamente utilizado no seu livro "Théorie analytique des probabilités". O método é relativamente simples e elementar, mas pouco conhecido. A sua aplicação se tem mostrado muito cômoda em equações encontradas pelo nosso colega Warwick Estevam Kerr nos seus estudos de Genética de Populações.

Nêste trabalho, que se destina especialmente aos geneticistas, não cogitaremos de dar às demonstrações todo o rigorismo lógico desejável em um trabalho de Matemática pura.

\section{2 - UM PROBLEMA TÍPICO DE GENÉTICA DE POPULAÇÕES}

Consideremos uma população onde se encontram indivíduos de constituição genética $\mathrm{AA}, \mathrm{Aa}$, aa, com frequências relativas respectivas uo, vo, wo. Se essa população se reproduz por autofecundação, e se $u_{n}, v_{n}, w_{n}$ indicam as frequências relativas na enésima geração, obtemos fàcilmente as equações :

$$
\mathrm{u}_{\mathrm{n}+1}=\underset{\mathrm{n}}{\mathrm{u}}+\frac{1}{4} \mathrm{v}_{\mathrm{n}}
$$$$
\mathrm{v}_{\mathrm{n}+1}=\frac{1}{2} \mathrm{v},
$$

$$
\mathrm{w}_{\mathrm{n}+\mathrm{l}}=\mathrm{w}_{\mathrm{n}}+\frac{1}{4} \mathrm{v} \text {. }
$$

Podemos procurar expressões algébricas apenas para un e $v_{n}$, pois temos 


$$
\mathbf{u}_{\mathbf{n}}+\mathbf{v}_{\mathbf{n}}+\mathbf{w}_{\mathbf{n}}=\mathbf{1}
$$

logo

$$
\mathbf{w}_{\mathbf{n}}=\mathbf{1}-\mathbf{u}-\mathbf{v}_{\mathbf{n}}
$$

A solução de $(2,2)$ é obtida fàcilmente por indução. Não seguiremos, porém, essa marcha, que falha inteiramente nos casos mais complexos.

Troquemos, em $(2,1), n$ por $n+1$ e obtemos

$$
\begin{aligned}
u_{n+2} & =u_{n+1}+\frac{-1}{4} v_{n+1} \\
& =u_{n+1}+\frac{1}{4} \cdot \frac{1}{2} v \text {, } \\
(2,4) \quad & u_{n+1}+\frac{1}{8} v_{n}
\end{aligned}
$$

As equações $(2,1)$ e $(2,4)$ nos permitem eliminar vn e obter a equação final

$$
2 u_{n+2}-3 u_{n+1}+u_{n}=0
$$

que nos cabe resolver.

\section{3 - UM PROBLEMA TIPICO DE PROBABILIDADES}

Consideremos uma sucessão de infinitas urnas, a primeira com uma bola preta e 5 brancas e cada uma das seguintes com duas pretas e 3 brancas. Um jogador retira uma bola da primeira urna e passa-a à segunda, retira uma da segunda e passa-a à terceira, e assim sucessivamente. Qual será a probabilidade de que seja preta a bola retirada da enésima urna ?

$\mathrm{Na}$ primeira urna a probabilidade de tirar uma bola preta é $\mathrm{p}_{\mathrm{o}}=\frac{1}{6}$ e a probabilidade de tirar bola branca é $\mathrm{q}_{\mathrm{o}}=\frac{5}{6}$. 
Uma vez conhecidas as probabilidades $p_{n} \cdot e q_{n}$ correspondentes à urna de ordem $n+1$, para a urna seguinte teremos

$$
\mathrm{p}_{\mathrm{n}+1}=\frac{1}{2} \mathrm{p}_{\mathrm{n}}+\frac{1}{3} \mathrm{q}
$$

pois há duas hipóteses favoráveis :

I) Tirar bola preta na urna de ordem $n+1\left(p_{n}\right)$ e bola preta na urna seguinte $\left(\frac{2+1}{5+1}=\frac{1}{2}\right)$;

II) Tirar bola branca na urna de ordem $n+1$ (q ) e bcla preta na urna seguinte $\left(\frac{2}{5+1}=\frac{1}{3}\right)$.

De $(3,1)$ obtemos logo

$$
\begin{gathered}
\mathrm{p}_{\mathrm{n}+1}=\frac{1}{2} \mathrm{p}_{\mathrm{n}}+\frac{\dot{1}}{3}\left(1-\mathrm{p}_{\mathrm{n}}\right) \\
\mathrm{p}_{\mathrm{n}+1}=\frac{1}{6} \mathrm{p}_{\mathrm{n}}+\frac{1}{3} .
\end{gathered}
$$

Se aí substituirmos $n$ por $n+1$ obteremos

$$
(3,3) \quad \cdots p_{n+2}=\frac{1}{6} p_{n+1}+\frac{1}{3} \text {. }
$$

A equação $(3,2)$ se pode subtrair de $(3,3)$ e então se chega à equação final

$$
\text { (3,4) } \quad 6 p_{n+2}-7 p_{n+1}+p_{n}=0,
$$

que nos cabe resolver. 


\section{4 - COMO SE APLICA O MÉTODO DAS FUNÇÕES}

\section{GERATRIZES}

Tomemos para exemplo de aplicação a equação

$$
2 u_{n+2}-3 u_{n+1}+u_{n}=0,
$$

que vimos atrás.

Admitamos a existência de uma função $f(x)$ que, desenvolvida em série, nos dê

$(4,2) f(x)=u_{0}+u_{1} x+u_{2} x^{2}+u_{3} x^{3}+\ldots+\underset{n}{u n}+\ldots$

Teremos então

$$
\begin{aligned}
& 2 f(x)=2 u_{0}+2 u_{1} x+2 u_{2} x^{2}+2 u_{3} x^{3}+\ldots, \\
& -3 x f(x)=\quad-3 u x-3 u x_{0}^{2}-3 u_{2} x^{3}-\ldots, \\
& x^{2} f(x)=\quad u_{0} x^{2}+u_{1} x^{3}+\ldots .
\end{aligned}
$$

A adição das três equações nos dá

$$
\begin{aligned}
& \left(x^{2}-3 x+2\right) f(x)=2 u+x(2 u-3 u)+ \\
& +x^{2}\left(2 u_{2}-3 u_{1}+u_{0}\right)+x^{3}\left(2 u_{3}-3 u_{2}+u_{1}\right)+ \\
& +\ldots+x^{n+2}\left(2 u_{n+2}-3 u_{n+1}+u_{n}\right)+\ldots
\end{aligned}
$$

Todos os coeficientes do segundo membro, com exceção dos dois primeiros, se anulam de acôrdo com $(4,1)$. E fica

$$
f(x)=\frac{0 u_{0}+x\left(2 u_{1}-3 u_{0}\right)}{x^{2}-3 x+2} .
$$


Suponhamos que, na população inicial, as frequências relativas eram

$$
\mathrm{u}_{\mathrm{o}}=0,2, \quad \mathrm{v}_{\mathrm{o}}=0,4, \quad \mathrm{w}_{\mathrm{o}}=0,4 .
$$

Temos então, de acôrdo com $(2,1)$,

$$
u_{1}=0,2+1 / 4 \cdot 0,4=0,3
$$

e fica

$(4,3)$

$$
f(x)=\frac{0,4}{x^{2}-3 x+2}
$$

Consideremos, de uma maneira mais geral, uma função

$$
f(x)=\frac{K}{a x^{2}+b x+c}
$$

e procuremos desenvolvê-la em série. Sendo $r$ e $s$ as raízes da equação $a x^{2}+b x+c=0$, temos

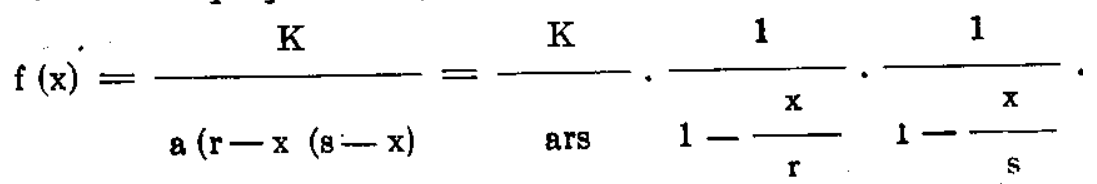

Mas

$$
\frac{1}{1-z}=1+z+z^{2}+z^{3}+\cdots,
$$

logo temos

$$
\begin{aligned}
& \frac{1}{1-\frac{x}{r}}=1+\frac{x}{r}+\frac{x^{2}}{2}+\frac{x^{3}}{3}+\ldots \\
& \frac{1}{1-\frac{x}{s}}=1+\frac{x}{s}+\frac{x^{2}}{2}+\frac{x^{3}}{3}+\cdots, \\
& s
\end{aligned}
$$


e portanto

$\left.(4,4) \mathrm{f}(\mathrm{x})=\frac{\mathrm{K}}{\mathrm{ars}} \mid \begin{array}{c}1+\mathrm{x}\left(\frac{1}{\mathrm{r}}+\frac{1}{\mathrm{~s}}\right)+\mathrm{x}\left(\mathrm{r}^{2}+\frac{1}{\mathrm{rs}}+\frac{1}{\mathrm{~s}^{2}}+\ldots+5\right. \\ \mathrm{n}\left(\frac{1}{\mathrm{r}^{\mathrm{n}}}+\frac{1}{\mathrm{r}^{\mathrm{n}-1} \mathrm{~s}}+\ldots+\frac{1}{\mathrm{~s}^{n}}\right)+\ldots\end{array}\right]$

A comparação de $(4,2)$ com 4,4$)$ nos mostra logo que

$$
\mathrm{u}_{\mathrm{n}}=\frac{\mathrm{K}}{\operatorname{ars}}\left(\frac{1}{\mathrm{r}^{\mathrm{n}}}+\frac{1}{\mathrm{r}^{\mathrm{n}-1} \mathrm{~s}}+\ldots+\frac{1}{\mathrm{~s}^{\mathrm{n}}}\right) .
$$

A expressão entre parênteses não é mais que uma soma dos termos de uma progressão geométrica de razão igual a $\frac{r}{s}$. logo temos

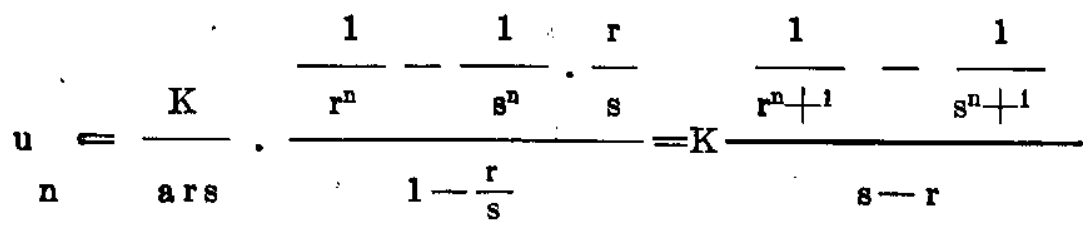

$$
\text { onde se supõe } 1-\frac{r}{s} \neq 0, \log 0 \mathrm{r} \neq \mathrm{s}
$$

No caso da função geratriz de $(4,3)$ temos $K=0,4, r=1$, $\mathrm{s}=2$, e portanto fica

$$
u_{n}=0,4\left(1-\frac{1}{2^{n+1}}\right) \text {. }
$$

Quando cresce o núméro de gerações, isto é, quando $\mathrm{n} \rightarrow \infty$, obtemos

$$
\lim _{\mathbf{n} \rightarrow \infty} \mathbf{u}_{\mathbf{n}}=0,4
$$

A marcha que acabamos de ver se aplica a qualquer equação linear homogênea de diferenças, isto é, do tipo 
${ }_{0}^{a}{ }_{n}{ }_{n-k}+a_{1}{ }_{n+k-1}+a_{2} u_{n k-2}+\ldots+a_{k} u_{n}=0$.

Quando a equação não é homogênea, é geralmente fàcil reduzí-la ao caso anterior.

Por exemplo a equação $(3,2)$, que não era homogênea, nos deu $(3,4)$, que já é homogênea.

Mas há um artifício geralmente mais vantajoso. Com efeito consideremos a equação

$(4,5)$

$$
\mathrm{p}_{\mathrm{n}+1}=\frac{1}{6} \mathrm{p}_{\mathrm{n}}+\frac{1}{3}
$$

e tomemos $\mathbf{p}_{\mathbf{n}}=\mathrm{y}_{\mathbf{n}}+\mathbf{a}$, onde a é uma constante a deter-

minar. Temos então $\mathbf{p}_{\mathbf{n}+1}=y_{\mathbf{n} \mid 1}+\mathbf{a}$ e $(4,5)$ nos dá

$$
y_{n+1}+a=\frac{1}{6} \quad y_{n}+\frac{1}{6} a+\frac{1}{3}
$$

logo

$$
\mathrm{y}_{\mathrm{n}+1}-\frac{1}{6} \mathrm{y}_{\mathrm{n}}=\frac{1}{3}-\frac{5}{6} \mathrm{a} .
$$

Basta impor a condição

$$
\begin{aligned}
& \frac{1}{3}-\frac{5}{6} \mathrm{a}=0, \\
& \log \mathrm{a}=\frac{2}{5}, \text { para obtermos a equação homogênea }
\end{aligned}
$$

$(4,6) \quad y_{n+1}-\frac{1}{6} y_{n}=0$.

E' facil verificar por indução que tôda equação do tipo

$$
\mathrm{t}_{\mathrm{n}+1}-\text { b. } \mathbf{t}_{\mathrm{n}}=0
$$


tem por soluçãọ

$$
\mathrm{t}_{\mathbf{n}}=\mathbf{t}_{\mathbf{0}} \cdot \mathrm{b}^{\mathbf{n}}
$$

Logo a solução de $(4,6)$ é

$$
\begin{aligned}
& \mathbf{y}_{\mathrm{n}}=\mathrm{y}_{0} \cdot\left(\frac{1}{6}\right)^{\mathrm{n}}: \\
& \mathbf{E} \text { como } \mathrm{p}_{\mathrm{n}}=\mathrm{y}_{\mathrm{n}}+\mathrm{a}=\mathrm{y}_{\mathrm{n}}+\frac{2}{5}, \text { fica } \\
& \mathrm{p}_{\mathbf{n}}=\mathrm{y}_{0}\left(\frac{1}{6}\right)^{\mathrm{n}}+\frac{2}{5} \\
&=\left(\mathrm{p}_{0}-\frac{2}{5}\right)\left(\frac{1}{6}\right)^{\mathrm{n}}+\frac{2}{5}: \\
&=\frac{2}{5}-\frac{7}{30}\left(\frac{1}{6}\right)^{n} .
\end{aligned}
$$

Conclui-se que, se $n \rightarrow \infty, p_{n} \rightarrow \frac{2}{5}$. Se interes-

sasse apenas êste limite, êle poderia ter sido obtido mais fàcilmente como se segue. Seja

$$
\mathrm{p}=\lim _{\mathrm{n} \rightarrow \infty} \mathrm{p}_{\mathrm{n}}=\lim _{\mathrm{n} \rightarrow \infty} \mathrm{p}_{\mathrm{n}+1}
$$

e $(3,2)$ nos dá

$$
p=\frac{1}{6} p+\frac{1}{3} \quad, \text { logo } p=\frac{2}{5} .
$$

Este é o limite procurado. 


\section{5 - MAIS UM EXEMPLO DE APLICAÇĀO FREQUENTE}

Uma equação de diferenças bastante frequente é

$$
\mathrm{p}_{\mathrm{n}+2}=\mathrm{p}_{\mathrm{n}}+\mathrm{p}_{\mathrm{n}+1}
$$

Dela obtemos logo

$$
f(x)=\frac{\left(p_{0}-p_{1}\right) x-p_{o}}{x^{2}+x-1} .
$$

As raízes do denominador são

$$
\mathrm{r}=\frac{-1+\sqrt{5}}{2} \quad, \quad \mathrm{~s}=\frac{-1-\sqrt{5}}{2} .
$$

Logo

$$
\begin{aligned}
& f(x)=(h x+j) \frac{1}{r-x} \cdot \frac{1}{s-x}, \\
& \quad \text { onde } h=p_{0}-p_{1}, j=-p_{0} .
\end{aligned}
$$

Obtemos entäo

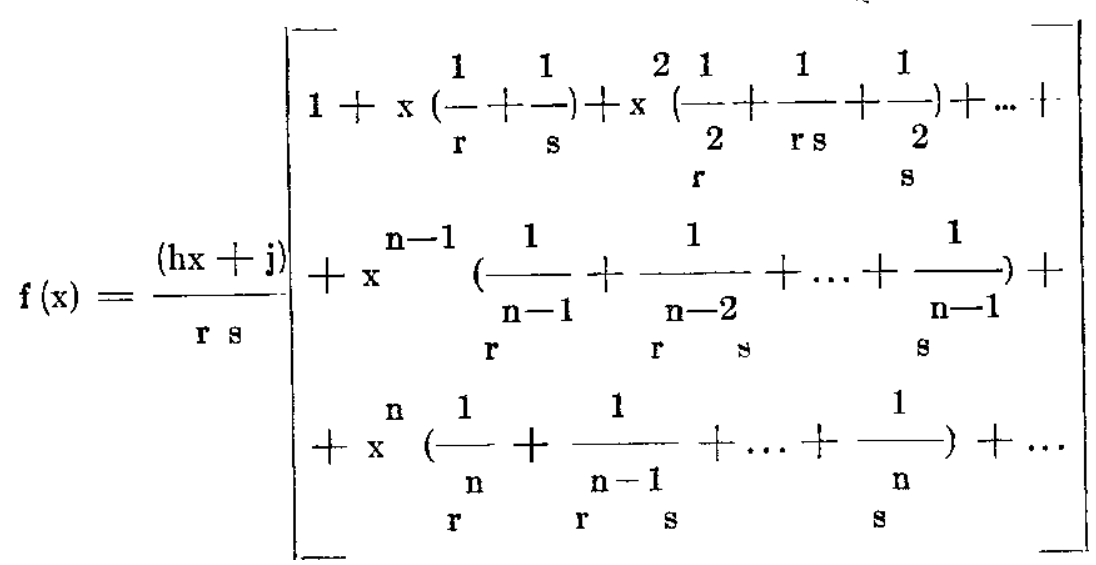




$$
\begin{aligned}
& p_{n}=\frac{j}{r s}\left(\frac{1}{n}+\frac{1}{n-1}+\ldots+\frac{1}{n}\right)+ \\
& +\frac{r}{r s}\left(\frac{1}{n-1}+\frac{1}{n-2}+\ldots+\frac{1}{n-1}\right) \\
& =j \frac{\frac{1}{r} s}{n+1}-\frac{1}{n+1} \cdot \frac{r}{r}-\frac{1}{n} \\
& s-r
\end{aligned}
$$

$\underset{\log 0}{\text { Suponhamos }} \mathrm{p}_{1}=1, \mathrm{p}_{1}=1$ e obtemos $\mathrm{h}=0, \mathrm{j}=-1$,

$$
\begin{aligned}
& p_{n}=\frac{2^{n}+1}{\sqrt{5}}\left[\frac{1}{\left(-1+\sqrt{5)^{n}+1}\right.}-\frac{1}{\left(-1-\sqrt{5)^{n}+1}\right.}\right] \\
& =\frac{1}{2^{n}+1} \sqrt{\overline{5}}\left[\left(1+\sqrt{5)^{n}+1}-\left(1-\sqrt{5}^{n}+1\right]\right.\right.
\end{aligned}
$$

Esta é a expressão do termo geral da sucessão

$1,1,2,3,5,8,13,21, \ldots$,

comumente denominada sucessão de Fibonacci, muito frequente na Genética de Populações.

\section{6 - BIBLIOGRAFIA CONSULTADA}

1 - USPENSKY, J. V. - Introduction to Mathematical Probability. McGraw-Hill, New York and London. 1937.

2 - KNOPF, Otto - Cálculo de Probabilidades. Labor, Barcelona - Buenos Aires. 1927.

3 - KERR, Warwick E. - Estudos sôbre a Genética de Populaçōes de Himenópteros em Geral e dos Apíneos Sociais em Particular - Piracicaba. 1950.

4 - DAVIS, Harold T. - The Theory of Linear Operators. The Principia Press, Bloomington, Indiana. 1936.

5 - HOGBEN, Lancelot - An Introduction to Mathematical Genetics. Norton \& Company, New York. 1946. 


$$
\text { , }
$$

